\title{
Second order evolution equations with parameter
}

\author{
by Jan Bochenek and Teresa Winiarska (Kraków)
}

\begin{abstract}
We give some theorems on continuity and differentiability with respect to $(h, t)$ of the solution of a second order evolution problem with parameter $h \in \Omega \subset \mathbb{R}^{m}$. Our main tool is the theory of strongly continuous cosine families of linear operators in Banach spaces.
\end{abstract}

1. Introduction. We consider the second order evolution problem

$$
\begin{aligned}
\frac{d^{2} u}{d t^{2}} & =A_{h} u+f(h, t), \quad t \in(0, T], \\
u(0) & =u_{h}^{0}, \\
u^{\prime}(0) & =u_{h}^{1},
\end{aligned}
$$

with parameter $h \in \Omega$, where $\left(A_{h}\right)_{h \in \Omega}$ is a family of linear (possibly unbounded) operators from a real Banach space $X$ into itself, $u$ is a mapping $\mathbb{R} \rightarrow X, f: \Omega \times \mathbb{R} \rightarrow X, \Omega$ is an open subset of $\mathbb{R}^{m}$, and $u_{h}^{0}, u_{h}^{1} \in X$ for $h \in \Omega$.

It is well known (see e.g. [1], [6]) that if $A_{h}$ is the infinitesimal generator of a strongly continuous cosine family $\left\{C_{h}(t): t \in \mathbb{R}\right\}$ of bounded linear operators from $X$ into itself, for $h \in \Omega$, and $f$ satisfies some regularity conditions, then the problem (1) has exactly one solution $u_{h}$ given by

(2) $u_{h}(t)=C_{h}(t) u_{h}^{0}+S_{h}(t) u_{h}^{1}+\int_{0}^{t} S_{h}(t-s) f(h, s) d s, \quad t \in[0, T], h \in \Omega$.

In (2), $S_{h}$, for $h \in \Omega$, is the operator sine function associated with $C_{h}$, defined by

$$
S_{h}(t) x:=\int_{0}^{t} C_{h}(s) x d s, \quad x \in X, t \in \mathbb{R}
$$

1991 Mathematics Subject Classification: 34K30, 35B30.

Key words and phrases: evolution problem, cosine family, evolution problem with parameter. 
The purpose of this paper is to present some theorems on continuity and differentiability with respect to $(h, t)$ of the solution of problem (1). Similar questions for the first order evolution problem are considered in [7]-[9].

2. Preliminaries. Assuming that $X, Y$ are Banach spaces we let $B(X, Y)$ be the Banach space of all bounded linear operators from $X$ to $Y$. If $X=Y$, then $B(X, X)$ is denoted by $B(X)$. The space of closed linear operators from $X$ into itself will be denoted by $C(X)$. For a given operator $A, D(A), R(A)$ and $P(A)$ will denote its domain, range and resolvent set, respectively.

Definition 1 (cf. [6]). Let $A_{h} \in C(X)$ with domain $D\left(A_{h}\right)=D_{h}$ for $h \in \Omega$. We call the family $\left(A_{h}\right)_{h \in \Omega} R$-continuous at $h_{0} \in \Omega$ if there exists a Banach space $Z$ and a family $T_{h} \in B(Z, X), h \in \Omega$, such that $h \in \Omega$,

(i) $R\left(T_{h}\right)=D_{h}$ and the mapping $Z \ni z \rightarrow T_{h} z \in D_{h}$ is bijective for all

(ii) the mappings $\Omega \ni h \rightarrow T_{h} \in B(Z, X)$ and $\Omega \ni h \rightarrow V_{h}=A_{h} T_{h} \in$ $B(Z, X)$ are continuous at $h_{0}$.

The continuity in $\Omega$ is defined to be the continuity at every point of $\Omega$.

We shall use the following simple lemma (cf. [7], Corollary 1).

Lemma 1. Let $A_{h} \in C(X)$ for $h \in \Omega$ and suppose $\lambda \in P\left(A_{h}\right)$ for all $h \in \Omega$. Then the mapping

$$
\Omega \ni h \rightarrow A_{h} \in C(X)
$$

is $R$-continuous at $h_{0} \in \Omega$ if and only if the mapping

$$
\Omega \ni h \rightarrow\left(\lambda-A_{h}\right)^{-1} \in B(X)
$$

is continuous at $h_{0}$.

Our main tool in this paper is the theory of strongly continuous cosine families of linear operators in Banach space. The basic ideas and results of this theory can be found for example in [6].

Recall that the infinitesimal generator of a strongly continuous cosine family $C(t)$ is the operator $A: X \supset D(A) \rightarrow X$ defined by

$$
A x:=\left.\left(d^{2} / d t^{2}\right) C(t) x\right|_{t=0}, \quad x \in D(A),
$$

where

(5) $D(A):=\{x \in X: C(t) x$ is twice continuously differentiable in $t\}$.

Let

$E:=\{x \in X: C(t) x$ is once continuously differentiable in $t\}$.

It is known (see [6], Proposition 2.2) that $D(A)$ is dense in $X$ and $A$ is a closed operator in $X$. 
If $A$ is the generator of $C(t)$, there exist constants $M \geq 1$ and $\omega \geq 0$ such that

$$
\|C(t)\| \leq M e^{\omega|t|} \quad \text { for } t \in \mathbb{R} .
$$

Moreover, let us notice (see [6], (2.17)-(2.19)) that

$$
\begin{gathered}
S(t) X \subset E \text { and } \quad S(t) E \subset D(A) \quad \text { for } t \in \mathbb{R}, \\
(d / d t) C(t) x=A S(t) x \quad \text { for } x \in E \text { and } t \in \mathbb{R}, \\
\left(d^{2} / d t^{2}\right) C(t) x=A C(t) x=C(t) A x \quad \text { for } x \in D(A) \text { and } t \in \mathbb{R} .
\end{gathered}
$$

The proof of the next propositions can be found in [2].

Proposition 1 (see [6]). Let $C(t), t \in \mathbb{R}$, be a strongly continuous cosine family in $X$ satisfying (6), and let $A$ be the infinitesimal generator of $C(t)$, $t \in \mathbb{R}$. Then, for $\operatorname{Re} \lambda>\omega, \lambda^{2}$ is in the resolvent set of $A$ and

$$
\lambda R\left(\lambda^{2} ; A\right) x=\int_{0}^{\infty} e^{-\lambda t} C(t) x d t \quad \text { for } x \in X,
$$

and

$$
R\left(\lambda^{2} ; A\right) x=\int_{0}^{\infty} e^{-\lambda t} S(t) x d t \quad \text { for } x \in X .
$$

Proposition 2. Under the assumptions of Proposition 1 , for $\operatorname{Re} \lambda>\omega$, $\lambda^{2}$ is in the resolvent set of $A$ and

$$
\left\|(d / d \lambda)^{k} \lambda\left(\lambda^{2}-A\right)^{-1}\right\| \leq \frac{M k !}{(\operatorname{Re} \lambda-\omega)^{k+1}} \quad \text { for } k=0,1, \ldots
$$

3. Assumptions and some helpful lemmas. Let $\left\{A_{h}\right\}_{h \in \Omega}$ be the family of linear operators defined in the Introduction. We make the following assumptions on $\left\{A_{h}\right\}_{h \in \Omega}$ :

$\left(\mathrm{Z}_{1}\right) \quad$ For each $h \in \Omega, A_{h}$ is the infinitesimal generator of a strongly continuous cosine family $\left\{C_{h}(t): t \in \mathbb{R}\right\}$ of bounded linear operators from $X$ into itself.

$\left(\mathrm{Z}_{2}\right) \quad$ The domain $D\left(A_{h}\right)=D$, for $h \in \Omega$, is independent of $h$ and the family $\left\{C_{h}(t)\right\}$ satisfies the inequality (6) with constants $M$ and $\omega$ independent of $h \in \Omega$.

Under assumptions $\left(\mathrm{Z}_{1}\right)$ and $\left(\mathrm{Z}_{2}\right)$, for each $h \in \Omega, A_{h}$ satisfies (9) with constants $M$ and $\omega$ independent of $h \in \Omega$.

In the sequel we shall need the following assumption.

( $\left.\mathrm{Z}_{3}\right) \quad$ There exist constants $M \geq 1$ and $\omega \geq 0$ independent of $h \in \Omega$ such that for $\operatorname{Re} \lambda>\omega, \lambda^{2}$ is in the resolvent set of $A_{h}$ and

$$
\left\|\lambda\left(\lambda^{2}-A_{h}\right)^{-1}\right\| \leq M(|\lambda|-\omega)^{-1} .
$$


The assumption (10) is stronger than the inequality resulting from (9) for $k=0$. Assumption $\left(\mathrm{Z}_{3}\right)$ has a technical character.

LEMMA 2. Suppose assumptions $\left(\mathrm{Z}_{1}\right)-\left(\mathrm{Z}_{3}\right)$ are satisfied. If the mapping

$$
\Omega \ni h \rightarrow A_{h} \in C(X)
$$

is $R$-continuous, then the mapping

$$
U \ni(\lambda, h) \rightarrow\left(\lambda^{2}-A_{h}\right)^{-1} \in B(X),
$$

where

$$
U:=\{(\lambda, h) \in \mathbb{C} \times \Omega: \operatorname{Re} \lambda>\omega\}
$$

is continuous.

Proof. Fix $\left(\lambda_{0}, h_{0}\right) \in U$ and let $(\lambda, h) \in U$. The assertion follows directly from the equality

$$
\begin{aligned}
& \left(\lambda^{2}-A_{h}\right)^{-1}-\left(\lambda_{0}^{2}-A_{h_{0}}\right)^{-1} \\
& \quad=\left(\lambda_{0}^{2}-\lambda^{2}\right)\left(\lambda^{2}-A_{h}\right)^{-1}\left(\lambda_{0}^{2}-A_{h}\right)^{-1}+\left(\lambda_{0}^{2}-A_{h}\right)^{-1}-\left(\lambda_{0}^{2}-A_{h_{0}}\right)^{-1} .
\end{aligned}
$$

THEOREM 1. Under the assumptions of Lemma 2, the mapping

$$
\Omega \times \mathbb{R} \ni(h, t) \rightarrow S_{h}(t) x \in X
$$

is continuous for each $x \in X$.

Proof. By assumption $\left(\mathrm{Z}_{1}\right)$, the formula (8) holds for each $h \in \Omega$ and $\operatorname{Re} \lambda>\omega$, i.e.

$$
R\left(\lambda^{2} ; A_{h}\right) x=\int_{0}^{\infty} e^{-\lambda t} S_{h}(t) x d t, \quad h \in \Omega, \operatorname{Re} \lambda>\omega, x \in X .
$$

A formal application of the inverse Laplace transform yields (cf. for example [4], p. 31)

$$
S_{h}(t) x=\frac{1}{2 \pi i} \int_{c-i \infty}^{c+i \infty} e^{\lambda t} R\left(\lambda^{2} ; A_{h}\right) x d \lambda, \quad t \in \mathbb{R}, h \in \Omega, x \in X,
$$

where $c>\omega$ is any constant, i.e. the line integral in (15) is taken along the straight line $\operatorname{Re} \lambda=c$. From (15) it follows that

$$
S_{h}(t) x=\frac{1}{2 \pi} \int_{-\infty}^{\infty} e^{(c+i \sigma) t}\left[(c+i \sigma)^{2}-A_{h}\right]^{-1} x d \sigma
$$

where $\lambda=c+i \sigma, \sigma \in(-\infty, \infty)$, is the path of integration in (15). By (10) we get

(17) $\left\|e^{(c+i \sigma) t}\left[(c+i \sigma)^{2}-A_{h}\right]^{-1} x\right\| \leq M e^{c t} \frac{1}{\sqrt{c^{2}+\sigma^{2}}\left(\sqrt{c^{2}+\sigma^{2}}-\omega\right)}\|x\|$. 
From (17) it follows that the improper integral in (16) is absolutely convergent uniformly in $(h, t) \in \Omega \times I$, where $I \subset \mathbb{R}$ is any bounded set.

Fix $\left(h_{0}, t_{0}\right) \in \Omega \times \mathbb{R}$, a compact neighborhood $K \subset \Omega \times \mathbb{R}$ of $\left(h_{0}, t_{0}\right)$ and an interval $[a, b] \subset \mathbb{R}$. By Lemma 2 the integrand in (16) is uniformly continuous in $K \times[a, b]$ as a function of $(h, t, \sigma)$. Therefore, using the well known theorem on the continuity of the improper integral with respect to parameters, we get the continuity of the mapping (14) at $\left(h_{0}, t_{0}\right)$. This completes the proof.

\section{LEMMA 3. If}

(i) the mapping $\Omega \ni h \rightarrow A_{h} \in C(X)$ is $R$-continuous,

(ii) the mapping $\Omega \times \mathbb{R} \ni(h, t) \rightarrow B_{h}(t) \in B(X)$ is continuous,

then the mapping

$$
\Omega \times \mathbb{R} \ni(h, t) \rightarrow B_{h}(t) A_{h}
$$

is R-continuous.

Proof. Fix $\left(h_{0}, t_{0}\right) \in \Omega \times \mathbb{R}$. By (i) there exist a Banach space $Z$ and operators $U_{h}, U_{h_{0}}, V_{h}, V_{h_{0}} \in B(Z, X)$ such that $U_{h}, U_{h_{0}}$ map $Z$ bijectively onto $D\left(A_{h}\right), D\left(A_{h_{0}}\right)$, respectively, $A_{h} U_{h}=V_{h}, A_{h_{0}} U_{h_{0}}=V_{h_{0}}$ and $\| U_{h}-$ $U_{h_{0}} \| \rightarrow 0$ and $\left\|V_{h}-V_{h_{0}}\right\| \rightarrow 0$ as $h \rightarrow h_{0}$.

Define $\widetilde{U}_{h}(t):=U_{h}$ and $\widetilde{V}_{h}(t):=B_{h}(t) V_{h}$. We have

$$
\left\|\widetilde{U}_{h}(t)-\widetilde{U}_{h_{0}}\left(t_{0}\right)\right\|=\left\|U_{h}-U_{h_{0}}\right\| \rightarrow 0
$$

and

$$
\begin{aligned}
\left\|\widetilde{V}_{h}(t)-\widetilde{V}_{h_{0}}\left(t_{0}\right)\right\|= & \left\|B_{h}(t) V_{h}-B_{h_{0}}\left(t_{0}\right) V_{h_{0}}\right\| \\
\leq & \left\|B_{h}(t)-B_{h_{0}}\left(t_{0}\right)\right\|\left\|V_{h}\right\| \\
& +\left\|B_{h_{0}}\left(t_{0}\right)\right\|\left\|V_{h}-V_{h_{0}}\right\| \rightarrow 0
\end{aligned}
$$

as $(h, t) \rightarrow\left(h_{0}, t_{0}\right)$.

On the other hand,

$$
\widetilde{V}_{h}(t)=B_{h}(t) V_{h}=B_{h}(t) A_{h} U_{h}=\left(B_{h}(t) A_{h}\right) \widetilde{U}_{h}(t)
$$

and

$$
\widetilde{V}_{h_{0}}\left(t_{0}\right)=B_{h_{0}}\left(t_{0}\right) V_{h_{0}}=B_{h_{0}}\left(t_{0}\right) A_{h_{0}} U_{h_{0}}=\left(B_{h_{0}}\left(t_{0}\right) A_{h_{0}}\right) \widetilde{U}_{h_{0}}\left(t_{0}\right) .
$$

Now the R-continuity of (18) follows from (19) and (20). The proof of Lemma 3 is complete.

THEOREM 2. Under the assumptions of Lemma 2 the mapping

$$
\Omega \times \mathbb{R} \ni(h, t) \rightarrow C_{h}(t) x \in X
$$

is continuous for each $x \in X$. 
Proof. From the known formula

$$
C(t+s)-C(t-s)=2 A S(t) C(s), \quad t, s \in \mathbb{R},
$$

(see [6], (2.23)), it follows that

(22) $\quad\left(C_{h}(t)-I\right) x=2 A_{h} S_{h}^{2}(t / 2) x, \quad t \in \mathbb{R}, h \in \Omega, x \in X$.

If $x \in D\left(A_{h}\right)=D$ we have

$$
\left(C_{h}(t)-I\right) x=2 S_{h}^{2}(t / 2) A_{h} x .
$$

Lemma 3 with $B_{h}(t):=2 S_{h}^{2}(t / 2)$, Theorem 1 , and (23) show the R-continuity of the mapping

$$
\Omega \times\left.\mathbb{R} \ni(h, t) \rightarrow C_{h}(t)\right|_{D} .
$$

On the other hand, by (6) and $\left(\mathrm{Z}_{2}\right), C_{h}(t): X \rightarrow X$ is a uniformly bounded operator for $h \in \Omega$ and $t \in[a, b]$, where $[a, b] \subset \mathbb{R}$ is any bounded interval. This gives the continuity of $(24)$ in the norm of $B(X)$ (see [3], p. 206). Using the Banach-Steinhaus theorem we obtain the assertion of Theorem 2 (cf. [4], p. 9).

4. Continuity with respect to a parameter. Let $\left(A_{h}\right)_{h \in \Omega}$ be a family of linear operators from $X$ into $X$ such that assumptions $\left(\mathrm{Z}_{1}\right),\left(\mathrm{Z}_{2}\right)$ are satisfied.

Lemma 4. Let $h_{0} \in \Omega$. If for any $x \in X$,

$$
\lim _{h \rightarrow h_{0}} C_{h}(t) x=C_{h_{0}}(t) x \quad \text { uniformly in } t \in[0, T]
$$

and the family $\left(A_{h}\right)_{h \in \Omega}$ is R-continuous at $h_{0}$, then

$$
\lim _{h \rightarrow h_{0}} C_{h}(t) x=C_{h_{0}}(t) x \quad \text { uniformly in }(t, x) \in[0, T] \times K,
$$

where $K$ is any compact subset of $X$.

The proof is the same as that of Proposition 1 in [7] with $\Phi(t, h)=$ $C_{h}(t)-C_{h_{0}}(t)$.

As a consequence of Lemma 4 we have

Corollary $1 . \lim _{h \rightarrow h_{0}} S_{h}(t) x=S_{h_{0}}(t) x$ uniformly in $[0, T] \times K$.

Proof. By (3) we have

$$
\left\|S_{h}(t) x-S_{h_{0}}(t) x\right\| \leq \int_{0}^{T}\left\|C_{h}(s) x-C_{h_{0}}(s) x\right\| d s .
$$

By (26), for any $\varepsilon>0$, there exists $\delta>0$ such that if $\left|h-h_{0}\right|<\delta$, then

$$
\left\|S_{h}(t) x-S_{h_{0}}(t) x\right\|<\varepsilon T \quad \text { for } t \in[0, T], x \in K .
$$


THEOREM 3. If the assumptions of Lemma 4 are satisfied, the mappings
(a) $\Omega \ni h \rightarrow u_{h}^{0} \in X$,
(b) $\Omega \ni h \rightarrow u_{h}^{1} \in X$,
(c) $f: \Omega \times[0, T] \rightarrow X$

are continuous and

(d) $f_{h}=f(h, \cdot):[0, T] \rightarrow X$

is $C^{1}$ for $h \in \Omega$, then for every $h \in \Omega$ there exists exactly one solution $u_{h}$ of the problem (1) and

$$
\lim _{h \rightarrow h_{0}} u_{h}(t)=u_{h_{0}}(t)
$$

uniformly in $t \in[0, T]$.

Pro of. By the assumptions, the solution of (1) is given by (2). Thus, by standard calculation we have

$$
\begin{aligned}
u_{h}(t)-u_{h_{0}}(t)= & \left(C_{h}(t)-C_{h_{0}}(t)\right) u_{h}^{0}+C_{h_{0}}(t)\left(u_{h}^{0}-u_{h_{0}}^{0}\right) \\
& +\left(S_{h}(t)-S_{h_{0}}(t)\right) u_{h}^{1}+S_{h_{0}}(t)\left(u_{h}^{1}-u_{h_{0}}^{1}\right) \\
& +\int_{0}^{t}\left[S_{h}(t-s)-S_{h_{0}}(t-s)\right] f_{h}(s) d s \\
& +\int_{0}^{t} S_{h_{0}}(t-s)\left[f_{h}(s)-f_{h_{0}}(s)\right] d s .
\end{aligned}
$$

Let $K$ be a compact neighborhood of $h_{0}$. Since the mappings (a), (b), (c) are continuous, the sets $K_{1}=\left\{u_{h}^{0}: h \in K\right\}, K_{2}=\left\{u_{h}^{1}: h \in K\right\}$ and $K_{3}=\left\{f_{h}(s): h \in K, s \in[0, T]\right\}$ are compact subsets of $X$. By Lemma 4 and Corollary 1 ,

$$
\left[C_{h}(t)-C_{h_{0}}(t)\right] u_{h}^{0} \stackrel{h \rightarrow h_{0}}{\longrightarrow} 0, \quad\left[S_{h}(t)-S_{h_{0}}(t)\right] u_{h}^{1} \stackrel{h \rightarrow h_{0}}{\longrightarrow} 0,
$$

and

$$
\left[S_{h}(t-s)-S_{h_{0}}(t-s)\right] f_{h}(s) \stackrel{h \rightarrow h_{0}}{\longrightarrow} 0,
$$

uniformly in $t, s \in[0, T]$. By assumption $\left(\mathrm{Z}_{2}\right)$ we have

$$
\left\|C_{h_{0}}(t)\left(u_{h}^{0}-u_{h_{0}}^{0}\right)\right\| \leq M e^{\omega T}\left\|u_{h}^{0}-u_{h_{0}}^{0}\right\| \stackrel{h \rightarrow h_{0}}{\longrightarrow} 0,
$$

uniformly in $t \in[0, T]$ and

$$
\|S(t) x\| \leq \int_{0}^{t} M e^{\omega s}\|x\| d s \leq \frac{M}{\omega}\left(e^{\omega t}-1\right)\|x\| .
$$

Therefore

$$
\left\|S_{h}(t)\right\| \leq \frac{M}{\omega}\left(e^{\omega T}-1\right) \quad \text { for } h \in \Omega .
$$


Hence

$$
\left\|S_{h_{0}}(t)\left(u_{h}^{1}-u_{h_{0}}^{1}\right)\right\| \stackrel{h \rightarrow h_{0}}{\longrightarrow} 0 \quad \text { and } \quad S_{h_{0}}(t-s)\left[f_{h}(s)-f_{h_{0}}(s)\right] \stackrel{h \rightarrow h_{0}}{\longrightarrow} 0,
$$

uniformly in $t, s \in[0, T]$. Thus the left hand side of (27) converges to zero, uniformly in $t \in[0, T]$.

COROLlARY 2. If the assumptions of Theorem 3 are satisfied for any $h_{0} \in \Omega$, then the mapping

$$
u: \Omega \times[0, T] \ni(h, t) \rightarrow u_{h}(t) \in X
$$

is continuous.

5. Differentiability with respect to a parameter. Let us recall (see [7], p. 223) the definition of differentiability of $\Omega \ni h \rightarrow A_{h}$.

Let $D$ be a normed vector space over $\mathbb{R}$ such that there exist a Banach space $Z$ and a bounded, linear, bijective mapping $T: Z \rightarrow D$. Setting $s B(D, Y)=\{A: D \rightarrow Y: A$ is linear and $A T \in B(Z, Y)\}$ we see that $s B(D, Y)$ is independent of $(Z, T)$.

Definition 2. Let $\Omega$ be an open subset of $\mathbb{R}$. A function $\Omega \ni h \rightarrow$ $A_{h} \in s B(D, Y)$ is said to be (continuously) differentiable at a point $h_{0} \in \Omega$ if there exist a Banach space $Z$ and a bounded, linear, bijective mapping $T$ : $Z \rightarrow D$ such that the mapping $\Omega \ni h \rightarrow A_{h} T \in B(Z, D)$ is (continuously) differentiable in the Fréchet sense.

In this case we put

$$
A_{h_{0}}^{\prime}=\left(\left.\frac{d}{d h} A_{h} T\right|_{h=h_{0}}\right) T^{-1} .
$$

The higher differentiability classes are defined in the standard manner.

Lemma 5. If $A u^{0}+f(0) \in D(A), A u^{1}+(d f / d t)(0) \in E, A$ is the generator of $C(t)$ and $f:[0, T] \rightarrow X$ is of class $C^{3}$ then the problem

$$
\begin{aligned}
& \frac{d^{2} u}{d t^{2}}=A u+f, \\
& u(0)=u^{0}, \\
& \frac{d u}{d t}(0)=u^{1},
\end{aligned}
$$

has exactly one solution which is of class $C^{4}$ in $[0, T]$.

P r o o f. It is well known that, under our assumptions, the solution of the problem (28) has the form

$$
u(t)=C(t) u^{0}+S(t) u^{1}+\int_{0}^{t} S(t-s) f(s) d s .
$$


Hence

$$
\begin{aligned}
\frac{d^{2} u}{d t^{2}}= & C(t)\left(A u^{0}+f(0)\right)+S(t)\left(A u^{1}+\frac{d f}{d t}(0)\right) \\
& +\int_{0}^{t} S(s) \frac{d^{2} f}{d t^{2}}(t-s) d s .
\end{aligned}
$$

Thus $w=d^{2} u / d t^{2}$ is the solution of the problem

$$
\begin{aligned}
& \frac{d^{2} w}{d t^{2}}=A w+\frac{d^{2} f}{d t^{2}}, \\
& w(0)=A u^{0}+f(0), \\
& \frac{d w}{d t}(0)=A u^{1}+\frac{d f}{d t}(0) .
\end{aligned}
$$

By Proposition 2.4 of [6] we conclude that $u$ is $C^{4}$ in $[0, T]$.

LEMMA 6. Suppose that the assumptions of Theorem 3 are satisfied at every $h_{0} \in \Omega$. If $A_{h} u^{0}+f_{h}(0) \in D, A_{h} u_{h}^{1}+\left(d f_{h} / d t\right)(0) \in E$ for $h \in \Omega$, $f_{h}=f(h, \cdot):[0, T] \rightarrow X$ is of class $C^{3}, d^{2} f / d t^{2}: \Omega \times[0, T] \rightarrow X$ is continuous and the mappings

$$
\Omega \ni h \rightarrow A_{h} u_{h}^{0}+f_{h}(0), \quad \Omega \ni h \rightarrow A_{h} u_{h}^{1}+\frac{d f_{h}}{d t}
$$

are continuous, then the mapping

$$
\Omega \times[0, T] \ni(h, t) \rightarrow \frac{d^{2} u_{h}}{d t^{2}}(t) \in X
$$

is continuous in $[0, T]$.

Lemma 6 is an immediate consequence of Lemma 5 and Theorem 3.

Now we prove

THEOREM 4. Let $\Omega$ be an open subset of $\mathbb{R}$ and suppose that assumptions $\left(\mathrm{Z}_{1}\right)-\left(\mathrm{Z}_{3}\right)$ hold. If

(1) the mappings $\Omega \ni h \rightarrow A_{h}, \Omega \ni h \rightarrow u_{h}^{0}$ are continuous in $\Omega$ and differentiable at $h_{0} \in \Omega$,

(2) $f_{h}:[0, T] \rightarrow f_{h}(t)$ is of class $C^{3}$ for $h \in \Omega$,

(3) the mappings

$$
\begin{aligned}
& \Omega \ni h \rightarrow A_{h} u_{h}^{0}+f_{h}(0) \\
& \Omega \ni h \rightarrow A_{h} u_{h}^{1}+\frac{d f_{h}}{d t}(0), \\
& \Omega \times[0, T] \ni(h, t) \rightarrow \frac{\partial f}{\partial h}(h, t)
\end{aligned}
$$

are continuous, 
(4) $A_{h} u_{h}^{0}+f_{h}(0) \in D$ and $A_{h} u_{h}^{1}+\left(d f_{h} / d t\right)(0) \in E$ for $h \in \Omega$,

then there exists exactly one solution $u_{h}(t)=u(h, t)$ of the problem (28) which is of class $C^{2}$ with respect to $t$ and differentiable with respect to $h$ at $h_{0}$.

Moreover,

$$
\lim _{h \rightarrow h_{0}} \frac{u_{h}(t)-u_{h_{0}}(t)}{h-h_{0}}=u_{h_{0}}^{\prime}(t),
$$

uniformly in $t \in[0, T]$, and $u_{h_{0}}^{\prime}$ is the solution of the problem

$$
\begin{aligned}
& \frac{d^{2} u_{h_{0}}^{\prime}}{d t^{2}}=A_{h_{0}} u_{h_{0}}^{\prime}+A_{h_{0}}^{\prime} u_{h_{0}}+f_{h_{0}}^{\prime}, \\
& u_{h_{0}}^{\prime}(0)=\left(u_{h_{0}}^{0}\right)^{\prime}, \\
& \frac{d u_{h_{0}}^{\prime}}{d t}(0)=\left(u_{h_{0}}^{1}\right)^{\prime} .
\end{aligned}
$$

Pr o of. We proceed similarly to the proof of Theorem 2 in [6]. For $h, h_{0}$ $\in \Omega$ we have

$$
\frac{d^{2}}{d t^{2}}\left(\frac{u_{h}-u_{h_{0}}}{h-h_{0}}\right)=A_{h}\left(\frac{u_{h}-u_{h_{0}}}{h-h_{0}}\right)+\frac{A_{h}-A_{h_{0}}}{h-h_{0}} u_{h_{0}}+\frac{f_{h}-f_{h_{0}}}{h-h_{0}}
$$

and

$$
\begin{aligned}
\frac{u_{h}(0)-u_{h_{0}}(0)}{h-h_{0}} & =\frac{u_{h}^{0}-u_{h_{0}}^{0}}{h-h_{0}}, \\
\frac{d u_{h}}{d t}(0)-\frac{d u_{h_{0}}}{d t}(0) & =\frac{u_{h}^{1}-u_{h_{0}}^{1}}{h-h_{0}} .
\end{aligned}
$$

If we take

$$
\begin{gathered}
F_{h}= \begin{cases}\frac{A_{h}-A_{h_{0}}}{h-h_{0}} u_{h_{0}}+\frac{f_{h}-f_{h_{0}}}{h-h_{0}} & \text { for } h \neq h_{0}, \\
A_{h_{0}}^{\prime} u_{h_{0}}+f_{h_{0}}^{\prime} & \text { for } h=h_{0},\end{cases} \\
v_{h}^{0}= \begin{cases}\frac{u_{h}^{0}-u_{h_{0}}^{0}}{h-h_{0}} & \text { for } h \neq h_{0}, \\
\left(u_{h_{0}}^{0}\right)^{\prime} & \text { for } h=h_{0},\end{cases} \\
v_{h}^{1}= \begin{cases}\frac{u_{h}^{1}-u_{h_{0}}^{1}}{h-h_{0}} & \text { for } h \neq h_{0}, \\
\left(u_{h_{0}}^{1}\right)^{\prime} & \text { for } h=h_{0},\end{cases}
\end{gathered}
$$


and

$$
v_{h}=\frac{u_{h}-u_{h_{0}}}{h-h_{0}} \quad \text { for } h \neq h_{0},
$$

then $v_{h}$, for $h \neq h_{0}$, is the solution of the problem

$$
\begin{aligned}
& \frac{d^{2} v_{h}}{d t}=A_{h} v_{h}+F_{h}, \\
& v_{h}(0)=v_{h}^{0}, \\
& \frac{d v_{h}}{d t}(0)=v_{h}^{1} .
\end{aligned}
$$

Therefore Theorem 4 will be proved if we can show that Theorem 3 can be applied.

Since the family $\left(A_{h}\right)_{h \in \Omega}$ and the mappings $\Omega \ni h \rightarrow v_{h}^{0} \in X, \Omega \ni h \rightarrow$ $v_{h}^{1} \in X$ satisfy all the assumptions of Theorem 3, we only have to prove that the mapping $\Omega \ni h \rightarrow F_{h}$ satisfies them as well. Taking $\lambda \in P\left(A_{h_{0}}\right)$ and $T=\left(A_{h_{0}}-\lambda I\right)^{-1}$ we have

$$
\frac{A_{h}-A_{h_{0}}}{h-h_{0}} u_{h_{0}}(t)=\left(\frac{A_{h}-A_{h_{0}}}{h-h_{0}} T\right) T^{-1} u_{h_{0}}(t) .
$$

Then, by Lemma $5, T^{-1} u_{h_{0}}$ is of class $C^{2}$ in $[0, T]$. This completes the proof.

Theorem 4 is the key to establishing theorems on higher regularity of the solution of (28) with respect to the parameter $h$.

\section{References}

[1] J. Bochenek, An abstract nonlinear second order differential equation, Ann. Polon. Math. 54 (1991), 155-166.

[2] H. O. Fattorini, Ordinary differential equations in linear topological spaces, I, J. Differential Equations 15 (1968), 72-105.

[3] T. Kato, Perturbation Theory for Linear Operators, Springer, New York, 1980.

[4] S. Krein, Linear Differential Equations in Banach Space, Amer. Math. Soc., 1972.

[5] M. Schechter, Differentiability of solutions of elliptic problems with respect to parameters, Boll. Un. Mat. Ital. A (5) 13 (1976), 601-608.

[6] C. C. Travis and G. F. Webb, Cosine families and abstract nonlinear second order differential equations, Acta Math. Acad. Sci. Hungar. 32 (1978), 75-96.

[7] T. Winiarska, Evolution equation with parameter, Univ. Iagell. Acta Math. 28 (1987), 219-227.

[8] —, Differential Equations with Parameter, Monograph 68, T. Kościuszko Technical Univ. of Cracow, 1988. 
[9] T. Winiarska, Parabolic equations with coefficients depending on $t$ and parameters, Ann. Polon. Math. 51 (1990), 325-339.

INSTITUTE OF MATHEMATICS

CRACOW UNIVERSITY OF TECHNOLOGY

WARSZAWSKA 24

31-155 KRAKÓW, POLAND

Reçu par la Rédaction le 15.12.1992

Révisé le 22.2.1993 